d'acide butyrique. Le boursouflement du fromage dans son vase en poterie était déjà un indice de cette fermentation butyrique mais non nécessairement, puisque les bactéries du groupe Coli pouvaient en être aussi la cause.

Nous mettons en parallèle dans le tableau qui suit : l'acidité $p \mathbf{H}$, l'acidité volatile exprimée en degrés Soxhlet et l'acidité exprimée en acide butyrique déterminée sur 100 grammes de fromage "Mich " de différents âges.

Age

$p \mathrm{H}$

Acidité volatile en degrés Soxhlet
Acidité volatile en gr. d'acide butyrique pour $100 \mathrm{gr}$. de fromage a Mich "

Gr.

46

60

80
1,01

1,32

1,76

Les espèces microbiennes isolées du "Mich» sont en premier lieu : Bacillus saccharobutyricus amylobacter (voir KLECKI-BREDEMANN) mis en évidence par examen microscopique du "Mich" et par cultures anaérobies; il est accompagné régulièrement de Streptococcus faecium (OrLA-Jensen). Suivant les échantillons de "Mich » examinés, on trouve aussi, mais non régulièrement: Bacillus mesentericus (TRevisan), Bacillus subtilis (СоHN), Proteus ichthyosmus (HAMMER), Flavobacterium butyri (BERGEY), etc.

En résumé, le "Kariche» subit la fermentation lactique, le "Mich" la fermentation butyrique à laquelle s'ajoutent encore l'action des diastases microbiennes et une certaine fermentation alcalinisante qui nous est révélée par le goût piquant du "Mich " et par la diminution de l'acidité $p \mathrm{H}$ avec l'âge et l'augmentation parallèle de l'acidité butyrique en degrés Soxhlet et en \%.

\title{
REVUE
}

\section{LES CONSTITUANTS MOINS CONNUS DU LAIT}

\author{
par G. GÉNIN \\ Ingénieur chimiste E. P. C.
}

Sous la présidence du $\mathrm{D}^{\mathrm{r}}$ G. Roche Lynch, président de la Society of Public Analysts, une réunion a été organisée, il y a quelques semaines à Londres, par le comité de cette société savante et la section des "produits alimentaires" de la Society of Chemicạl Industry, dans le but d'étudier les constituants les moins connus du lait. A cette occasion, plusieurs communications furent présentées par le Professeur T. P. Hilditch, M. R. T. Twigg, le Professeur H. D. Kay, le Dr E. C. V. Mattick, le Dr S. J. Folley, le Dr J. H. 
Bushill, le Dr L. H. Lampitt, M. D. F. Filmer, M. E. B. Anderson et le $\mathrm{D}^{\mathrm{r}} \mathrm{R}$. MacWalter.

\section{Des constituants secondaires du lait.}

Le Professeur T. P. Hilditch a étudié "quelques constituants secondaires de la matière grasse du lait et leur rôle probable". Il a constaté que sur 100 molécules d'acides gras combinées sous la forme de triglycérides dans la matière grasse du lait de vache il y a environ 27 à 35 molécules d'acide oléique et 24 à 27 molécules d'acide palminique, tandis que les acides butyrique, myristique et stéarique représentent ensemble 7 à 10 molécules. Les autres acides, qui sont assez peu définis, constituent ce qu'on appelle les constituants secondaires : on a trouvé de l'acide laurique, puis de l'acide eaproïque et des acides caprylique et caprique.

On a vérifié également dans la matière grasse du lait la présence d'une faible proportion (généralement nettement inférieure à $1 \%$ ) d'acides polyéthénoïdes contenant 20 et même 22 atomes de carbone dans leur molécule.

Le fait que la proportion de glycérides entièrement saturés est anormalement élevée, par rapport à ce qu'on constate dans les autres graisses naturelles, a conduit l'auteur et ses collaborateurs à penser que dans la matière grasse du lait, les glycérides des acides gras inférieurs se forment à partir des oléoglycérides pré-formés par un phénomène combiné d'oxydation et de réduction.

Lorsque de l'huile de foie de morue est ajoutée à la nourriture des vaches laitières, certains des acides polyéthénoïdes caractéristiques en $\mathrm{C}^{20}$ et en $\mathrm{C}^{22}$ de cette huile, passent dans la matière grasse du beurre, qui peut alors contenir jusqu'à 5 ou $7 \%$ de ces acides. En même temps le rendement en matière grasse du lait diminue et sa composition est profondément modifiée. La proportion des glycérides inférieurs est réduite à environ le tiers de sa valeur habituelle, la proportion de glycérides myristique, palmilique et stéarique est également réduite, par contre, celle d'oléoglycérides n'est que peu modifiée. Ces modifications ne sont d'ailleurs que temporaires et lorsque l'animal cesse de recevoir de l'huile de foie de morue, son rendement en matière grasse et la composition de cette dernière redeviennent normaux.

\section{Phénomènes d'oxydation réduction.}

M. R. S. TWIGG a étudié la capacité que possèdent le lait cru et le lait chauffé de réduire une certaine quantité de solution de bleu de méthylène de concentration standard. Cette étude qui a porté sur du lait entier et sur du lait écrémé présente une grande importance depuis que l'essai au bleu de méthylène est de plus en plus utilisé pour apprécier la qualité bactériologique du lait. L'auteur a opéré 
sur des laits à très faible teneur en bactéries et dans des conditions qui interdisaient le développement des bactéries au cours des essais, ces derniers étant effectués en l'absence d'oxygène.

Des résultats observés, il résulte que le lait possède naturellement, même s'il ne contient pas de bactéries, le pouvoir de réduire le bleu de méthylène. Par contre, la séparation de la crème entraîne l'élimination de substances réductrices et d'enzymes et le lait écrémé ne réduit plus le bleu de méthylène. Le chauffage à $63^{\circ} \mathrm{C}$. pendant une demi-heure et la pasteurisation du lait réduisent totalement ou partiellement l'activité des enzymes. Le lait écrémé pasteurisé se comporte comme le lait entier pasteurisé, mais avee une activité réductrice réduite. La crème elle-même non chauffée, et mise en suspension dans l'eau, réduit le bleu de méthylène. La pasteurisation du lait, ou le chauffage à une température plus élevée réduit son activité.

On a étudié un grand nombre de substances réductrices en vue d'examiner le pouvoir qu'elles ont de réduire le bleu de méthylène en présence de crème. De toutes celles examinées, seules les aldéhydes et l'hypoxanthine ont une activité réductrice très nette. Des essais ont été effectués en vue de séparér par dialyse les constituants réducteurs du lait entier ou de la crème non lavée. Aucun résultat satisfaisant n'a été obtenu et la dialyse rendrait certains enzymes inactifs. Des expériences récentes ont enfin montré que l'acide ascorbique du lait ne jouerait qu'un rôle insignifiant dans la réduction du bleu de méthylène dans des conditions anaérobiques.

\section{Lipase et phosphatase.}

Le Professeur H. D. Kay, le Dr E. C. V. Mattick et le Dr S. J. Foldey ont présenté une étude sur quelques récents travaux concernant la lipase et la phosphatase du lait de vache. Après avoir décrit les méthodes de dosage de la phosphatase, les auteurs ont souligné les difficultés que l'on rencontre dans la détermination de la lipase du lait.

Les meilleurs résultats sont ceux qu'on obtient en utilisant la tributyrine associée avec un sel tampon spécial d'un $p \mathbf{H}$ de 8,5 , avec contrôle bactériologique avant et après dosage, l'acide butyrique obtenu par hydrolyse étant séparé par distillation, puis titré. Les conditions relatives au mode opératoire, par exemple l'agitation, doivent être rigoureusement standardisées.

On a constaté que tous les laits crus contiennent de la lipase en quantité variable suivant l'animal et au cours de la période de lactation. La lipase du lait est plus rapidement détruite que la phosphatase. Il y a plus de lipase dans le sérum du lait que dans la matière grasse, l'inverse s'applique à la phosphatase. Il est probable qu'il 
n'y a pas de relation entre la lipase et la phosphatase du lait, la mesure de la teneur en phosphatase constituant un indice d'efficacité de la glande mammaire.

\section{Le dosage de la catalase.}

M. E. B. Anderson et le Dr R. J. McWalter ont constaté, au cours de leurs travaux sur les enzymes du lait, qu'il était indispensable de procéder à une étude des méthodes que l'on a proposées pour le dosage de la catalase. Ces méthodes se divisent en deux groupes principaux : dosage volumétrique de l'oxygène dégagé de l'eau oxygénée ou dosage volumétrique du peroxyde non décomposé, ces deux dosages s'effectuant directement par le permanganate de potassium ou indirectement par la détermination de l'iode libéré de l'iodure de potassium.

Après étude comparative des différentes méthodes, les auteurs ont proposé un mode opératoire nouveau qui évite certaines difficultés signalées par divers expérimentateurs. Ce dosage ne peut être d'ailleurs qu'empirique, car les substances contenues dans l'échantillon détruisent les enzymes et il faut opérer dans des conditions toujours standards.

Nous citerons enfin pour terminer l'étude présentée par le Dr J. H. Bushild, le $D^{r}$ L. H. Lampist et M. D. F. Filmer sur nos connaissances actuelles concernant quelques constituants azotés secondaires du lait.

\section{BIBLIOGRAPHIE ANALYTIQUE}

\section{LES LIVRES}

Clunie Harvey (W.) et Hill(H.). - Produits laitiers. 1 vol. relié toile $1 / 2$ in octavo, 387 pages, 73 illustrations. Prix : $16 \mathrm{sh}$. net. H. K. Lewis et Co. Ltd, Londres, 1937.

Les A., qui sont tous deux inspecteurs sanitaires, ont rédigé un manuel d'hygiène pratique donnant sous une forme suffisamment coneise les divers usages du lait, ainsi que les méthodes de fabrication et les méthodes de contrôle. Les méthodes de fabrication sont rédigées d'une façon très claire et les appareils soigneusement décrits. La législation anglaise relative à chacun des produits étudiés a été, bien entendu, convenablement résumée, et les questions sanitaires relatives à chaque produit remarquablement étudiées.

Le premier chapitre traite de la crème glacée (ice-cream). La définition de ce produit est assez difficile, en raison des nombreux produits qui entrent dans sa fabrication. Toutefois, l'Association des Fabricants d'ice-cream de Grande-Bretagne et d'Irlande a proposé que l'ice-cream soit définie comme suit : un produit glacé ne contenant pas moins de $8 \%$ de matière grasse de beurre et de $18 \%$ d'extrait sec dégraissé de lait. Les A. reconnaissent qu'en 